\title{
Almacenamiento de carbono en sistemas agroforestales en los Llanos Orientales de Colombia
}

\author{
Héctor Eduardo Hernández Núñez ${ }^{1,2 *}$, Hernán J. Andrade ${ }^{3}$, Juan Carlos Suárez Salazar ${ }^{1}$, \\ José R. Sánchez A. ${ }^{1}$, David R. Gutiérrez S. ${ }^{1}$, Gustavo Adolfo Gutiérrez García ${ }^{1}$, \\ Edwin Trujillo Trujillo ${ }^{1} \&$ Fernando Casanoves ${ }^{4}$ \\ 1. Grupo de Investigación en Agroecosistemas y Conservación en Bosques Amazónicos, Universidad de la Amazonia, \\ Caquetá, Colombia; heduardohn@gmail.com (*Correspondencia),juansuarez1@gmail.com,jr.sanchez@udla.edu.co, \\ da.gutierrez@udla.edu.co,g.gutierrez@udla.edu.co,botanico_ua@yahoo.com \\ 2. Universidad del Tolima, Facultad de Agronomía, Doctorado en Ciencias Agrarias, Ibagué-Tolima, Colombia. \\ 3. Grupo de Investigación Producción Ecoamigable de Cultivos Tropicales, Universidad del Tolima, Facultad de \\ Ingeniería Agronómica, Tolima, Colombia; hjandrade@ut.edu.co \\ 4. Centro Agronómico Tropical de Investigación y Enseñanza, Turrialba 30501, Costa Rica; casanoves@catie.ac.cr
}

$$
\text { Recibido 16-VII-2020. Corregido 15-XI-2020. Aceptado 16-XII-2020. }
$$

\begin{abstract}
Carbon storage in agroforestry systems in Colombia's Eastern Plains. Introduction: Coffee, cocoa crops and pastures for livestock are agricultural activities of economic interest in Colombia. When these activities are developed under agroforestry systems (AFS), they promote conservation and increase carbon fixation and, therefore, climate change mitigation. Objective: The study estimated carbon storage in aboveground biomass, necromass and soil organic carbon under SAF with cocoa (SAF cocoa), SAF with coffee (SAF coffee), silvopastoral systems (SPS) and forest in Mesetas, Meta (Colombia). Methods: Forty-four sampling plots were established, where dasometric measurements were taken from individuals with a trunk diameter at breast height ( $\mathrm{dbh}) \geq 2.5 \mathrm{~cm}$ (saplings, trees and large trees), whose values were transformed to carbon with biomass models and a default carbon fraction. In the three agricultural systems, the number of cocoa and coffee trees and associated plants was counted, and the type of use (timber, food, combustion) was identified. Results: Carbon storage showed significant differences $(\mathrm{P}<0.0001)$ among land uses. The highest accumulation was found in forest, with $216.6 \mathrm{t} \mathrm{C}^{-1}$, exceeding in 59, 72 and $73 \%$ to SAF cocoa, SSP and SAF coffee, respectively. The botanical families Fabaceae, Lauraceae and Primulaceae presented the greatest carbon storage. In SAF cocoa, the greatest accumulation of carbon was found in species for human food; in SAF coffee and SSP, the greatest storage was presented by timber species. Conclusion: These results highlight the potential for carbon storage in the most important productive systems in the Meta department, which is important for designing strategies that allow for integrating actions to mitigate greenhouse gas emissions and to promote the local peasant economy.
\end{abstract}

Key words: forests; climate change; greenhouse effect; agriculture; livestock; mitigation.

Hernández Núñez, H.E., Andrade, H.J., Suárez Salazar, J.C., Sánchez A., J.R., Gutiérrez S., D.R., Gutiérrez García, G.A., Trujillo Trujillo, E., \& Casanoves, F. (2021). Almacenamiento de carbono en sistemas agroforestales en los Llanos Orientales de Colombia. Revista de Biología Tropical, 69(1), 352-368. DOI 10.15517/rbt. v69i1.42959

El cambio climático es uno de los mayores desafíos que enfrenta la humanidad en la actualidad (Arias \& Rosales, 2019). Este es un fenómeno global y creciente, que genera impactos negativos socioeconómicos y afecta la salud pública, la disponibilidad del recurso hídrico y la productividad agroindustrial (Arteaga \& Burbano, 2018). El principal responsable del cambio climático es la creciente emisión de gases de efecto invernadero (GEI), i.e. dióxido 
de carbono - $\mathrm{CO}_{2}^{-}$, metano $\mathrm{CH}_{4}$ - y óxido nitroso - $\mathrm{N}_{2} \mathrm{O}$ - (López et al., 2018; Kuosmanen, Zhou, $\&$ Dai, 2020).

Los GEI, resultado de acciones antropogénicas, han causado un desequilibrio en el planeta, alterando de diversas formas el balance de radiación del sistema superficie-atmósfera (Buitrago, Ospina, \& Narváez, 2018; Olorunfemi, Komolafe, Fasinmirin, \& Olufayo, 2019). Dentro de los causantes del incremento de las emisiones de GEI, se encuentra el cambio de uso de la tierra (Olorunfemi et al., 2019), particularmente la conversión de bosques a tierras agrícolas, la cual es una práctica común en los trópicos (Asase \& Tetteh, 2015). Estos bosques están especialmente amenazados por prácticas de tala y quema para el establecimiento de cultivos o pasturas (Nijmeijer, Lauri, Harmand, \& Saj, 2018; Jiménez, Fonseca, \& Pazmiño, 2019). Esta práctica es responsable del 33.3 $\%$ del total de emisiones de GEI (Arteaga \& Burbano, 2018); mientras que, dentro de las actividades agropecuarias, la ganadería es el mayor aportante con un $14.5 \%$ del total (Gerber, Steinfeld, \& Henderson, 2013; Rojas, Nejadhashemi, Harrigan, \& Woznicki, 2017).

A pesar de este panorama, la concentración de GEI en la atmósfera puede reducirse a través de la reducción de emisiones de $\mathrm{CO}_{2}$ y la creación e incremento de sumideros de carbono (IPCC, 2019). Varios estudios han demostrado que los bosques tropicales contribuyen a regular la concentración de $\mathrm{CO}_{2}$ en la atmósfera (Yepes et al., 2015; Hurtado, Corte, \& Triana, 2017; Segura, Andrade, \& Mojica Sánchez, 2019), a través de la fijación en su biomasa y en el suelo (Paipa \& Triana, 2018; Segura, Andrade, \& Sierra, 2020). Por esta razón, muchos países tropicales aspiran a proteger los bosques para cumplir los objetivos de las políticas de mitigación de la biodiversidad y el clima (Sullivan et al., 2017), tal como los proyectos REDD+ (Nielsen, 2016).

Los sistemas agroforestales (SAF) también pueden contribuir a la mitigación del cambio climático mediante el secuestro y almacenamiento de carbono (Albrecht \& Kandji, 2003; Andrade \& Ibrahim, 2003; Andrade, Brook, \&
Ibrahim, 2008; Soto-Pinto, Anzueto, Mendoza, Ferrer, \& de Jong, 2010). Según Villa et al. (2020) los SAF son los sistemas alimentarios sostenibles más importantes del mundo, y permiten la integración de árboles y otros cultivos. Esta diversificación de la producción permite obtener mayores beneficios sociales, económicos y ambientales (Asase \& Tetteh, 2015). Entre los principales beneficios que prestan los SAF se incluyen la recuperación, conservación y mejora de la biodiversidad, el aumento de las reservas de carbono, la fijación biológica de nitrógeno y el ciclaje de nutrientes, la disminución de la erosión y el mantenimiento de la fertilidad del suelo (Villa et al., 2020). En consecuencia, los SAF son soluciones potenciales para los esfuerzos que articulan la conservación de la biodiversidad y el incremento de la productividad agrícola (Wartenberg et al., 2017), ya que pueden ser más rentables y productivos que las prácticas de monocultivo convencionales (Villa et al., 2020).

En Colombia, entre las principales actividades agropecuarias se encuentran la ganadería, la caficultura y la cacaocultura (Baena, 2019; Gutiérrez, Gutiérrez-Montes, Hernández-Núñez, Suárez, \& Casanoves, 2020), las dos últimas se realizan en un 40 y $90 \%$ en SAF, respectivamente (Espinosa, 2016; Zapata, 2019). En el caso de la ganadería, Colombia avanza en la implementación sistemas silvopastoriles (SSP) (Arciniegas \& Flórez, 2018; Díaz, Enciso, Triana, Muriel, \& Burkart, 2018). De esta forma, las actividades agropecuarias desarrolladas bajo SAF permiten la captura de carbono, por lo que deben tener atención como estrategias para mitigar el cambio climático (Olorunfemi et al., 2019). Por lo tanto, la estimación de la capacidad de almacenamiento de carbono se constituye en una labor fundamental que permite generar estrategias de conservación y uso sostenible de los bosques y las unidades agrícolas (Andrade \& Ibrahim, 2003; Andrade, Segura, Feria, \& Suárez, 2016; Hurtado et al., 2017).

El presente estudio estima la acumulación de carbono en bosques, SAF con café, SAF cacao y SSP en el departamento del Meta, 
Colombia. Las preguntas que guiaron la presente investigación fueron: ¿Cómo varía la acumulación de carbono en los sistemas de producción agropecuaria de mayor importancia en el departamento del Meta? ¿Qué compartimentos dentro de los sistemas de producción presentan la mayor acumulación de carbono? y ¿Cuánto es el aporte en carbono de las especies acompañantes de uso maderable, alimenticio y combustible? Estas estimaciones permitirán generar acciones en la planificación de proyectos de captura de carbono para mitigar el cambio climático para promover sistemas de producción sostenibles.

\section{MATERIALES Y MÉTODOS}

Área de estudio: El estudio se realizó en zona rural del municipio de Mesetas, departamento del Meta, Colombia ( $3^{\circ} 23^{\prime} 03^{\prime \prime} \mathrm{N} \&$ $\left.74^{\circ} 02^{\prime} 43^{\prime \prime} \mathrm{W}\right)$. El clima de este municipio es templado húmedo, con temperatura media anual entre 22 y $24^{\circ} \mathrm{C}$, precipitaciones entre 2 500 y $3000 \mathrm{~mm}$ año ${ }^{-1}$, con 150 a 200 días de lluvia y humedad relativa media anual de 80 a $85 \%$ (IDEAM, 2017).

Establecimiento de parcelas: Se establecieron 44 parcelas de muestreo, distribuidas en igual número en bosque, SAF con cacao (SAF cacao), SAF con café (SAF café) y SSP. Los bosques correspondieron a la categoría "Bosque denso alto de tierra firme" (IDEAM, 2010); las parcelas fueron establecidas en áreas de bosque mayor a 3 ha y con mínima intervención humana. Las parcelas en SAF cacao y SAF café fueron establecidas en cultivos con más de 3.5 años de establecidos, que contaran con un área mínima de 1 ha; en los SAF café renovados (soqueados), se eligieron los cultivos en los que la práctica de renovación se hubiera realizado con dos años de anticipación; en el caso de SAF cacao, fueron cultivos con un mínimo de 70 árboles de cacao por parcela. Las parcelas de SSP fueron establecidas en lotes mayores a 3 ha.

En bosque, se siguió la metodología del Inventario Forestal Nacional (IDEAM, 2018); mientras que en los SAF y SSP se siguió lo propuesto por Andrade \&Ibrahim (2003) y Yepes et al. (2011). En el caso del bosque, se estableció una unidad de muestreo (UMS), la cual se conformó por cinco parcelas principales de muestreo anidadas y concéntricas, ubicadas en forma de cruz con una central, cada una con una distancia de $80 \mathrm{~m}$ entre centroides (Fig. 1A). Cada parcela principal fue de $15 \mathrm{~m}$ de radio y en ella se midieron los fustales grandes (FG; diámetro a la altura del pecho -dap- $\geq 30$ $\mathrm{cm})$. En las subparcelas, de $7 \mathrm{~m}$ de radio, se midieron los fustales (F; dap $\geq 10 \mathrm{~cm} \mathrm{y}<30$ $\mathrm{cm}$ ); mientras que en las subparcelas de $3 \mathrm{~m}$ de radio se midieron los latizales ( $\mathrm{L}$; dap $\geq 2.5$ $\mathrm{cm}$ y $<10 \mathrm{~cm})$. En los SAF con cacao y café, se establecieron parcelas de $1000 \mathrm{~m}^{2}(20 \times 50$ m) (Fig. 1B) y en los SSP las parcelas fueron circulares también de $1000 \mathrm{~m}^{2}$ (Fig. 1C) cada una en el centro del lote, en la cual se tomaron las medidas dasométricas en árboles con dap $\geq$ 2.5 (Yepes et al. 2011).

En cada UMP, se caracterizó la composición florística y la estructura arbórea (Jagoret et al., 2017). Para esto, se contó el número de árboles de cacao, café y plantas asociadas (L, F y FG), las cuales fueron identificadas a nivel de especie; también, se identificaron los individuos muertos en pie (Suárez, Ngo Bieng, Melgarejo, Di Rienzo, \& Casanoves, 2018). Las colectas botánicas para la identificación de especies fueron realizadas usando la metodología usada por Ricker (2019). Con base en estas variables, se calculó la riqueza, y los índices de Shannon-Weaver y de Simpson a nivel de especie. Además de los diámetros, se tomaron las siguientes medidas dasométricas: área de copa, altura total y altura comercial si correspondía (Ngo Bieng et al., 2013; Saj, Jagoret, \& Todem, 2013; Andrade et al., 2016; Olorunfemi et al., 2019). Con esta variables se calculó el área de copa y área basal (Suárez et al., 2018).

Carbono arriba de suelo: Este componente se conformó por los árboles (forestal), árboles de cacao/café, madera caída, hojarasca y pasto. El carbono forestal, compuesto por los latizales, fustales y fustales grandes y el carbono de árboles de café y cacao se estimó 

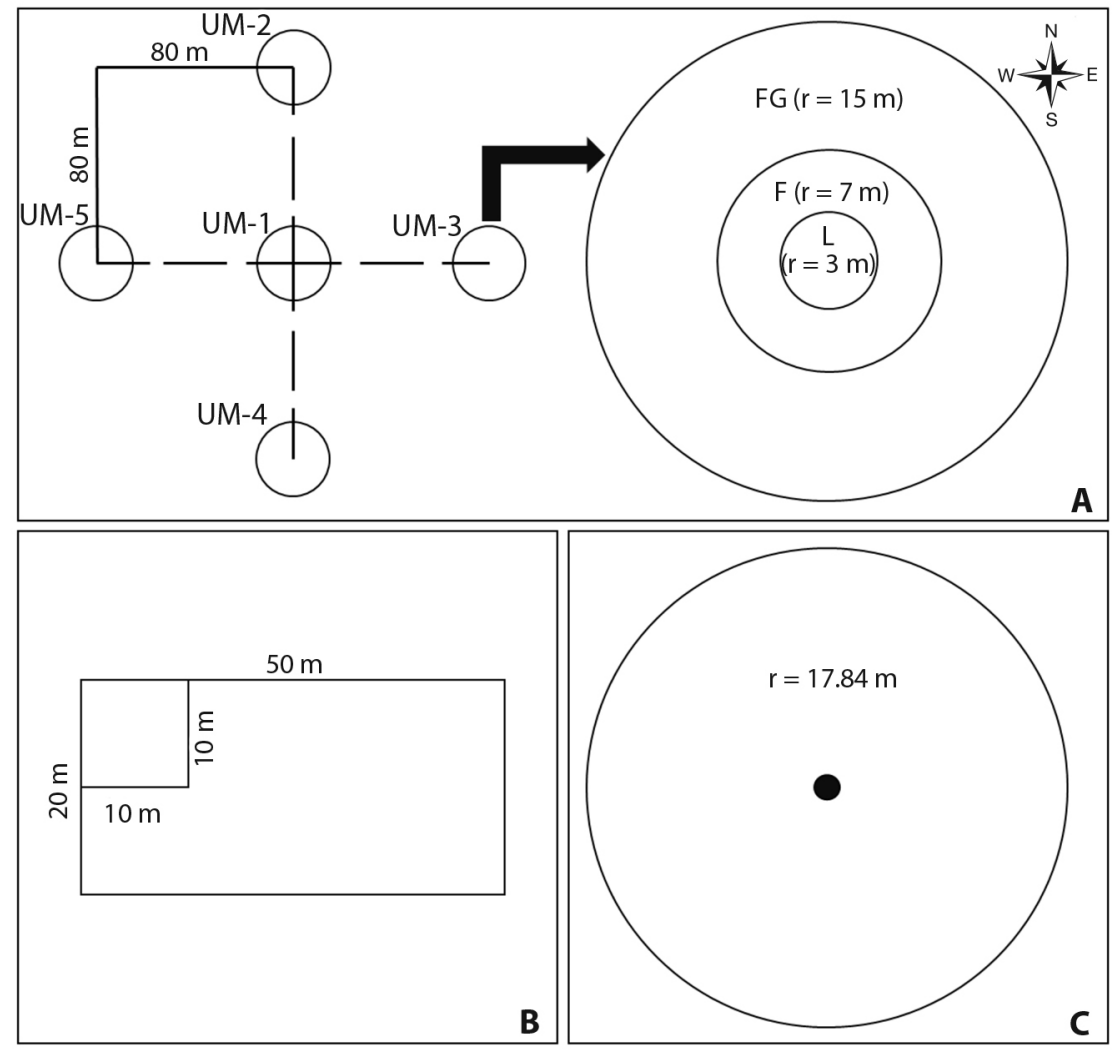

Fig. 1. Esquema de establecimiento de parcelas para medición de carbono en: A. Bosques, B. Sistemas agroforestales de cacao y café y C. Sistemas silvopastoriles en el municipio de Mesetas, Meta, Colombia.

Fig. 1. Scheme for the establishment of plots for carbon measurement in: A. Forests, B. Agroforestry systems of cocoa and coffee and C. Silvopastoral systems in the municipality of Mesetas, Meta, Colombia.

empleando modelos de biomasa (Tabla 1). Para reducir las incertidumbres en la estimación de carbono, se seleccionaron modelos específicos para Theobroma cacao L., Coffea arabica L. y palmas (Arecaceae); mientras que para las especies forestales se seleccionó un modelo multiespecie desarrollado en la misma zona de vida, tal como lo recomiendan el IPCC (2000) y Casanoves, Cifuentes, \& Chacón (2017). En los tres sistemas productivos se discriminó el carbono acumulado en las especies forestales de acuerdo con su categoría de uso: alimento humano, maderable, combustible y "otro uso".

La necromasa se dividió en madera muerta (en pie y caída) y hojarasca. La materia seca de los árboles muertos en pie se estimó mediante los modelos de biomasa arriba mencionados y se les aplicó el factor de corrección de 0.5, el cual fue empleado por Casanoves et al. (2017), para considerar su grado de descomposición. Las mediciones en madera caída fueron realizadas en las piezas finas (diámetro $\geq 2 \mathrm{~cm} \mathrm{y}<$ $20 \mathrm{~cm}$ ) y gruesas (diámetro $\geq 20 \mathrm{~cm}$ ), a cada pieza se le midió el diámetro y la dureza con ayuda del penetrómetro dinámico, siguiendo las recomendaciones de Yepes et al (2001). En bosque, el muestreo fue realizado en la UMS-2 y UMS-4, en cada UMS se realizaron cuatro transectos en forma de cruz siguiendo los puntos cardinales, cada uno de $30 \mathrm{~m}$, en donde fueron medidas las piezas gruesas ubicadas en el transecto (IDEAM, 2018). Estos transectos se dividieron en tres secciones (10 m cada una) y las piezas finas se midieron en el último metro 
TABLA 1

Modelos alométricos de biomasa empleados para estimar la biomasa arriba del suelo de árboles en diferentes usos del suelo en el municipio de Mesetas, Meta, Colombia

TABLE 1

Alometric biomass models used to estimate the above-ground biomass of trees in different land uses in the municipality of Mesetas, Meta, Colombia

\begin{tabular}{lcc}
\multicolumn{1}{c}{ Especies } & Modelo & Fuente \\
Árboles de café & $\mathrm{Ba}=0.36-0.18 * \mathrm{~d}_{15}+0.08 * \mathrm{~d}_{15}{ }^{2}$ & $\begin{array}{c}\text { Andrade et al. (2016) } \\
\text { Árboles de cacao }\end{array}$ \\
Árboles (multiespecie) & $\mathrm{Ba}=0.202 * \mathrm{~d}_{50} 2.112$ & Smiley et al. (2008) \\
Palmas & $\mathrm{Ba}=\mathrm{D} \times \exp (-1.499+2.148 \times \operatorname{Ln}(\mathrm{dap})+0.207 \times$ & Chave et al. (2005) \\
\hline
\end{tabular}

Ba: biomasa arriba del suelo $\left(\mathrm{kg}_{\text {individuo }}{ }^{-1}\right) ; \mathrm{d}_{15}$ : diámetro del tronco a una altura de $15 \mathrm{~cm} ; \mathrm{d}_{50}$ : diámetro del tronco a una altura de $50 \mathrm{~cm}$; dap: Diámetro del tronco a la altura del pecho $(\mathrm{cm})$; D: Densidad de madera $\left(\mathrm{g} \mathrm{cm}^{-3}\right)$.

$\mathrm{Ba}$ : above ground biomass ( $\mathrm{kg}$ individual $\left.{ }^{-1}\right) ; \mathrm{d}_{15}$ : diameter of the trunk at $15 \mathrm{~cm}$ height of; $\mathrm{d}_{50}$ : diameter of the trunk at 50 $\mathrm{cm}$ height; dap: diameter of the trunk at breast height $(\mathrm{cm})$; D: Wood density $\left(\mathrm{g} \mathrm{cm}^{-3}\right)$.

de cada una de estas secciones (IDEAM, 2018). En los SAF, los cuatro bordes de la subparcela de $10 \times 10 \mathrm{~m}$ (Fig. 1B) se utilizaron como transectos lineales para medir las piezas ubicadas en ellas. La materia seca de la madera caída fue calculada a través de las siguientes ecuaciones.

La densidad de la madera se estimó con base en la resistencia a la penetración, de acuerdo con la siguiente ecuación (Yepes et al., 2011):

$$
\rho=0.6451-0.388(\log (P))-0.377 \times I+0.30(\log (P)) I
$$

donde: $\rho$ : densidad de la pieza $\left(\mathrm{g} \mathrm{cm}^{-3}\right)$; P: penetración (cm); I = 0 , si $\mathrm{P} \leq 1 ; \mathrm{I}=1$, si $\mathrm{P}>1$

El valor de $\mathrm{P}$ se calculó realizando 20 golpes al penetrómetro. Si el penetrómetro no entró por completo luego de ese número de golpes, la longitud obtenida debe ser dividida en 20; si el penetrómetro entró por completo en la pieza en menos de 20 golpes, la longitud de penetración $\mathrm{P}$ se calcula como:

$$
P=\frac{20}{(g-0.5)}
$$

donde $g$ es el número de golpes hasta penetrar por completo.
El volumen de las piezas de madera caída se estimó con base en la ecuación recomendada por el IPCC (2000):

$$
V i=\frac{\pi^{2}}{8 L} \sum d i_{i}^{2}
$$

donde: Vi: volumen de la pieza i $\left(\mathrm{m}^{3}\right)$; L: longitud del transecto $(\mathrm{m})$; di: diámetro de la pieza i (m)

Con estas dos variables, se estimó la materia seca de la madera caída:

$$
M=\sum(V i \times \rho i)
$$

donde: M: materia seca de madera caída (g); Vi: volumen de la pieza i $\left(\mathrm{m}^{3}\right)$; $\rho$ i: densidad de la pieza $\mathrm{i}\left(\mathrm{g} \mathrm{cm}^{-3}\right)$.

El muestreo de hojarasca comprendió todos los residuos orgánicos vegetales sobre la superficie del suelo, incluyendo hojas, frutos, semillas y ramas con diámetro $<2 \mathrm{~cm}$. Dentro de cada UMP en SAF cacao, SAF café y SPP, se ubicaron tres marcos de $0.5 \times 0.5 \mathrm{~m}$ cada uno, en los cuales se colectó y pesó la hojarasca acumulada en fresco y se tomó una submuestra de cerca $500 \mathrm{~g}$, la cual fue secada a $70{ }^{\circ} \mathrm{C}$, hasta 
obtener un peso constante (Yepes et al., 2011) y así convertir los pesos frescos a materia seca.

La estimación de carbono del pasto en SPP se realizó a través de la técnica de aforos, la que consistió en realizar muestreos destructivos en tres marcos de $4 \mathrm{~m}^{2}$ por parcela (Yepes et al., 2011). En cada uno de los marcos, se cortó toda la biomasa a nivel del suelo, pesándola en fresco y tomando muestra para materia seca. $\mathrm{La}$ estimación de carbono en biomasa y necromasa se obtuvo multiplicando la biomasa o necromasa por 0.47 (IPCC, 2006).

Carbono en suelo: En cada parcela, se estimó la concentración de carbono orgánico en suelos (COS) y la densidad aparente (DA), a una profundidad de $0-20 \mathrm{~cm}$, debido a que esta capa es la más importante para el almacenamiento de carbono en estos suelos (Delgado, Rangel, \& Silva, 2018). En SAF cacao, SAF café y SSP, se tomó una muestra de suelo compuesta por submuestras tomadas en puntos al azar; mientras que en el caso de los bosques fueron tomadas a $2 \mathrm{~m}$ de distancia con un azimut de $45^{\circ}$ desde el centro de cada UMS (IDEAM, 2018). La estimación de la concentración de COS se realizó con el método de titulación de Walkey y Black (IGAC, 2006). La DA se estimó con el método cilindro (Lizcano et al., 2017), tomando cuatro muestras en SAF cacao, SAF café y SSP y cinco muestras en el bosque.

Análisis de datos: Se realizaron análisis de varianza para estimar las diferencias en el almacenamiento de carbono entre los cuatro usos de suelo. Los supuestos de normalidad y homogeneidad de varianzas fueron evaluados usando Shapiro-Wilks y gráficos de residuos versus predichos, respectivamente. Las diferentes variables fueron analizadas usando el modelo lineal general (Di Rienzo, Macchiavelli, \& Casanoves, 2011) y las comparaciones fueron realizadas con la prueba de LSD de Fisher (P $<0.05)$. Se calcularon las incertidumbres de la estimación de carbono total para cada sistema a partir de intervalos de confianza (95\%). Los análisis estadísticos se realizaron en el software estadístico InfoStat (Di Rienzo et al., 2019).

\section{RESULTADOS}

\section{Almacenamiento de carbono en los usos} de suelos: El almacenamiento de carbono total presentó diferencias significativas $(\mathrm{P}<0.0001)$ entre los usos del suelo, siendo el bosque el de mayor valor (216.6 $\left.\mathrm{t} \mathrm{C} \mathrm{ha}^{-1}\right)$, superando en 59 $\%$ a SAF cacao y $72-73 \%$ a SAF café y SSP (Tabla 2). La acumulación de carbono arriba del suelo en el bosque fue estadísticamente superior $(\mathrm{P}<0.0001)$ a lo encontrado en los otros usos el suelo, superándolos en 53, 74 y $69 \%$ al SAF cacao y SAF café y SSP, respectivamente (Tabla 2). El carbono acumulado que consideró solo los árboles de sombra en los tres sistemas y se detectaron diferencias significativas $(\mathrm{P}<0.0001)$ entre usos del suelo, siendo superior en bosque (158.7 $\left.\mathrm{t} \mathrm{C} \mathrm{ha}^{-1}\right)$, lo que resultó un 82-85\% superior a SAF cacao, SAF café y SSP. Un comportamiento diferente ocurrió en el COS, que fue mayor en SAF café y bosque (42.8 y $40.8 \mathrm{t} \mathrm{C} \mathrm{ha}^{-1}$, respectivamente), los cuales no presentaron diferencias significativas $(p>0.05)$ entre sí, pero sí fueron superiores estadísticamente $(\mathrm{P}<0.0001)$ que SSP y SAF cacao, que contenían 35.4 y 28.7 t $\mathrm{C} \mathrm{ha}^{-1}$, respectivamente (Tabla 2).

En los cuatro usos del suelo, la mayor acumulación del carbono arriba del suelo se encontró en el componente forestal y representó el $89,94,51$ y $48 \%$ en bosque, SSP, SAF café y SAF cacao, respectivamente. En SAF cacao y SAF café, el mayor aporte al carbono forestal lo generaron los FG, con 66 y $68 \%$, respectivamente. Por el contrario, la mayor acumulación del carbono forestal en el bosque fueron los $\mathrm{F}$. En SAF cacao y SAF café, el carbono acumulado en los cultivos (árboles de cacao y café) representó el 48-51 \% del carbono arriba de suelo, no obstante, los árboles de cacao acumularon $85 \%$ más carbono que los de café, siendo esta diferencia significativa $(\mathrm{P}<0.0001)$ (Tabla 2). El carbono en necromasa fue mayor en el bosque $\left(19.3 \mathrm{t} \mathrm{C} \mathrm{ha}^{-1}\right)$ y presentó diferencias estadísticas $(\mathrm{P}<0.0001)$ respecto a SAF cacao 
y SAF café. El $25 \%$ de carbono en necromasa en bosque, correspondió a arboles muertos en pie; mientras que en SAF cacao, SAF café y SPP no se encontró este componente.

Los SSP presentaron la mayor acumulación de COS (59\% del total), la cual fue mayor al carbono forestal (39\%) (Tabla 2). El $74 \%$ del carbono total en SAF café, fue encontrado en el suelo, el cual presentó el mayor reservorio después del componente forestal (Tabla 2). En SAF cacao, el COS fue menor que en los demás usos del suelo, este representó el $33 \%$ del carbono total; sin embargo, fue superior al carbono forestal (Tabla 2).

Carbono forestal por familias botánicas en sistemas productivos: En SAF cacao, SAF café y SSP se encontraron especies de 15, 12 y 9 familias taxonómicas, respectivamente (Fig. 2). En SAF cacao y SPP, el 84-86 \% del carbono forestal lo acumularon el 43-44 \% de las familias; en cambio, en SAF café, el 82 $\%$ del carbono forestal fue acumulado por el $25 \%$ de las familias más dominantes (Fabaceae, Lauraceae y Bignoniaceae) (Fig. 2). Las Fabaceae acumularon el mayor porcentaje de carbono forestal en SAF café y SSP (46 y 42 $\%$, respectivamente). Las especies de la familia Lauraceae presentaron la mayor acumulación de carbono en SAF cacao (24\% del carbono forestal) y la segunda mayor en SAF café. Persea americana Mill. y Ocotea tomentosa van der Werff. fueron las especies de Lauraceae que presentaron mayor almacenamiento de carbono en SAF cacao y SAF café, respectivamente. La familia Primulaceae presentó la segunda mayor acumulación de carbono en SAF cacao y SPP, 14 y $27 \%$ del carbono forestal, respectivamente.

TABLA 2

Stock de carbono arriba del suelo, necromasa y carbono orgánico del suelo en diferentes usos de suelos en el municipio de Mesetas, Meta, Colombia

TABLE 2

Above ground Carbon stock, necromass and organic carbon in different soil uses in the municipality of Mesetas, Meta, Colombia

\begin{tabular}{|c|c|c|c|c|c|}
\hline \multirow{2}{*}{ Componente } & Bosque & SAF Cacao & SAF Café & SSP & \multirow{2}{*}{$P$ valor } \\
\hline & \multicolumn{4}{|c|}{${\mathrm{t} \mathrm{C} \mathrm{ha}^{-1}}$} & \\
\hline Forestal & $156.4 \pm 8.6 \mathrm{a}$ & $28.5 \pm 4.9 b$ & $7.7 \pm 3.0 \mathrm{c}$ & $23.1 \pm 4.1 \mathrm{bc}$ & $<0.0001$ \\
\hline Latizales & $8.9 \pm 0.70 \mathrm{a}$ & $0.02 \pm 0.02 b$ & $0.03 \pm 0.2 b$ & & $<0.0001$ \\
\hline Fustales & $90.1 \pm 4.9 \mathrm{a}$ & $9.6 \pm 2.2 b$ & $2.39 \pm 0.6 b$ & $2.42 \pm 0.6 b$ & $<0.0001$ \\
\hline Fustales grandes & $57.5 \pm 6.72 \mathrm{a}$ & $18.9 \pm 3.8 b$ & $5.24 \pm 3.1 \mathrm{~b}$ & $20.7 \pm 4.1 b$ & $<0.0001$ \\
\hline Cultivo & & $24.7 \pm 2.1 \mathrm{a}$ & $3.6 \pm 0.8 \mathrm{a}$ & & $<0.0001$ \\
\hline Necromasa & $19.3 \pm 2.1 \mathrm{a}$ & $6.5 \pm 1.9 b$ & $3.6 \pm 0.48 b$ & & $<0.0001$ \\
\hline Muertos en Pie & $4.8 \pm 0.8$ & & & & \\
\hline Madera caída & $14.5 \pm 1.9 \mathrm{a}$ & $4.4 \pm 1.9 b$ & $1.91 \pm 0.5 b$ & & 0.0001 \\
\hline Hojarasca & & $2.1 \pm 0.2 \mathrm{a}$ & $1.71 \pm 0.2 \mathrm{a}$ & & 0.5687 \\
\hline Pastura & & & & $1.4 \pm 0.1$ & \\
\hline Arriba del suelo & $175.7 \pm 9.5 \mathrm{a}$ & $59.8 \pm 4.5 b$ & $15.0 \pm 3.1 \mathrm{c}$ & $24.5 \pm 4.17 \mathrm{c}$ & $<0.0001$ \\
\hline Carbono orgánico del suelo & $40.8 \pm 1.5 \mathrm{a}$ & $28.8 \pm 4.1 \mathrm{c}$ & $42.8 \pm 9.5 \mathrm{a}$ & $35.4 \pm 1.6 b$ & $<0.0001$ \\
\hline Carbono total & $216.6 \pm 9.1 \mathrm{a}$ & $88.6 \pm 5.0 \mathrm{~b}$ & $57.8 \pm 9.8 \mathrm{c}$ & $60.0 \pm 3.9 \mathrm{~d}$ & $<0.0001$ \\
\hline $\begin{array}{l}\text { Incertidumbre en la estimación } \\
\text { de carbono total }(\%)\end{array}$ & 8 & 11 & 33 & 12 & \\
\hline
\end{tabular}

Los valores representan la media \pm error estándar. Valores de $\mathrm{P}<0.05$ indican que hay diferencias significativas en el Stock de carbono entre usos de suelo; letras diferentes muestran las diferencias entre los de usos de suelos.

The values represent the mean \pm standard error. P-value $<0.05$ indicates that there are significant differences in Carbon Stock between land uses; different letters show the differences between land uses. 


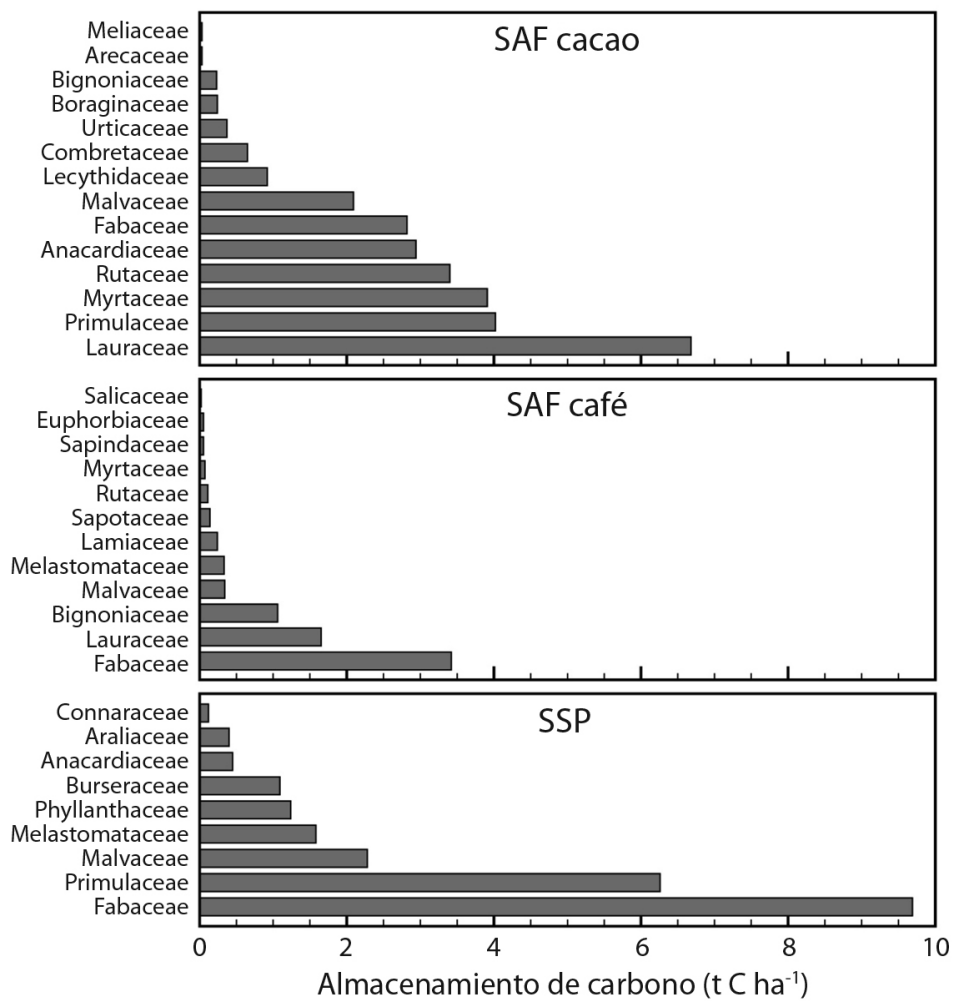

Fig. 2. Distribución del almacenamiento de carbono forestal por familias botánicas en sistemas agroforestales (SAF) con cacao, SAF con café y sistemas silvopastoriles (SSP) en el municipio de Mesetas, Meta, Colombia.

Fig. 2. Distribution of forest carbon storage by botanical families in agroforestry systems (AFS) with cocoa, AFS with coffee and silvopastoral systems (SP) in the municipality of Mesetas, Meta, Colombia.

Relación entre almacenamiento de carbono y uso de las especies forestales: En SAF cacao, las especies usadas como alimento humano presentaron el mayor reservorio del carbono forestal (38\%), lo cual fue 61-63\% superior a las empleadas como combustible y madera (Fig. 3). Además, las especies de uso alimenticio de los SAF cacao acumularon $97 \%$ más carbono a aquellas que brindan el mismo servicio en SAF café. En contraste, los SSP no presentaron especies que suplan las necesidades de alimento para humanos. La mayor acumulación de carbono forestal en SAF café y SSP se encontró en las especies maderables (69 y $53 \%$, respectivamente). No obstante, los SPP capturaron $57 \%$ más carbono en especies maderables que los SAF café (Fig. 3). El carbono en especies de uso combustible fue la segunda mayor en los tres usos de suelo, con el 22, 14 y $39 \%$ del carbono forestal en SAF cacao, SAF café y SPP, respectivamente.

Se encontraron 50 especies que capturan carbono y a la vez brindan algún producto a las familias productoras: 23 maderable, 18 combustible, 7 alimento y 2 alimento y madera. En SAF cacao y SSP, el mayor número de especies son maderables, 12 y 7, respectivamente; en cambio, en SAF café, la mayoría de las especies (ocho) sirven como combustible y seis eran maderables. Solo en SAF café se encontraron especies con doble servicio (alimento humano y maderable); y en el SAF cacao se detectaron más especies con uso alimenticio que en SAF café: seis y tres especies, respectivamente. Persea americana Mill., Psidium guajava L. y Citrus $x$ sinensis (L.) 

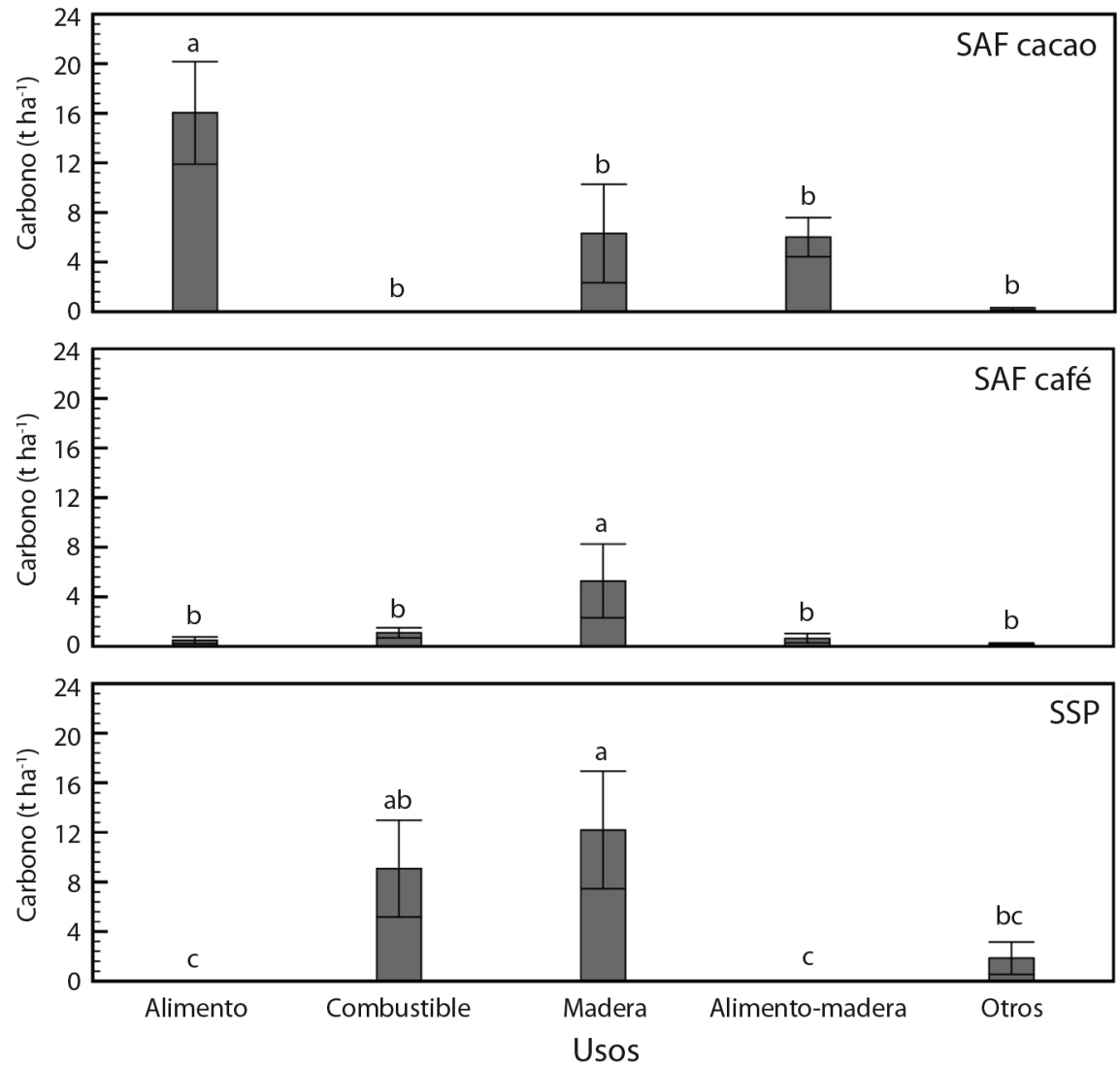

Fig. 3. Almacenamiento de carbono por uso de las especies del componente forestal en sistemas agroforestales (SAF) con cacao, SAF con café y sistemas silvopastoriles (SSP) en el municipio de Mesetas, Meta, Colombia. Las barras representan los valores medios ( \pm error estándar) y letras distintas muestra las diferencias $(\mathrm{P}<0.05)$ entre los tipos de uso de especies. Fig. 3. Carbon storage by use of forest species in agroforestry systems (AFS) with cocoa, AFS with coffee, and silvopastoral systems (SP) in the municipality of Mesetas, Meta, Colombia The bars represent the mean values $( \pm$ standard error) and different letters show the differences $(\mathrm{P}<0.05)$ between species use types.

Osbeck, producen alimento para humanos, y presentaron la mayor acumulación de carbono en SAF cacao; las dos primeras especies también estuvieron presente en los SAF café, pero acumulando mucho menos carbono (Tabla 3). En SAF café, una especie perteneciente al género Inga y Ocotea tomentosa van der Werff. y Parkia cf. nitida Miq., todas maderables, acumularon el mayor carbono (21-28\% del carbono de las especies maderables y 17-22\% del carbono forestal) (Tabla 3). En SSP, la especie maderable Ormosia nobilis Tul. dominó el almacenamiento de carbono, aportando el 31 $\%$ del carbono forestal. En SAF cacao, Acacia mangium Willd. logró la mayor acumulación de carbono entre las especies maderables, pero fue un $82 \%$ menor que lo almacenado en $\mathrm{Per}$ sea americana Mill (Tabla 3).

\section{DISCUSIÓN}

La acumulación de carbono fue diferente entre usos del suelo; diversos autores afirman que existe la acumulación de carbono tiene alta dependencia de la estructura arbórea y la composición florística (Asase \& Tetteh, 2015; Andrade, Segura, Canal, Huertas, \& Mosos, 2017; Paipa \& Triana, 2018; 
TABLA 3

Almacenamiento de carbono en especies forestales de acuerdo con el uso doméstico o comercial en sistemas agroforestales (SAF) cacao, SAF café y sistemas silvopastoriles (SSP) en Mesetas, Meta, Colombia

TABLE 3

Carbon storage in forest species according to domestic or commercial use in agroforestry systems (AFS) cocoa, AFS coffee and silvopastoral systems (SP) in Mesetas, Meta, Colombia

\begin{tabular}{|c|c|c|c|c|c|}
\hline \multirow{2}{*}{ Familia } & \multirow{2}{*}{ Nombre Científico } & \multirow{2}{*}{ Uso } & SAF Cacao & SAF Café & SSP \\
\hline & & & \multicolumn{3}{|c|}{ t C ha-1 } \\
\hline \multirow[t]{2}{*}{ Anacardiaceae } & Mangifera indica Willd. & Alimento & 1.93 & & \\
\hline & Tapirira sp 1 & Madera & 1.01 & & 0.45 \\
\hline Arecaceae & Bactris gasipaes Kunth. & Alimento & 0.03 & & \\
\hline \multirow[t]{3}{*}{ Bignoniaceae } & Handroanthus barbatus (E. Mey.) Mattos. & Madera & 0.02 & & \\
\hline & Jacaranda copaia (Aubl.) D. Don. & & & 1.06 & \\
\hline & Tabebuia rosea (Bertol.) DC. & & 0.21 & & \\
\hline Boraginaceae & Cordia alliodora Kunth ex Steud. & & 0.24 & & \\
\hline \multirow[t]{2}{*}{ Burseraceae } & Crepidospermum sp 1 & & & & 0.87 \\
\hline & Trattinnickia rhoifolia Willd. & & & & 0.22 \\
\hline Combretaceae & Terminalia amazonia (J.F. Gmel.) Exell. & & 0.65 & & \\
\hline \multirow{11}{*}{ Fabaceae } & Inga edulis Mart. & Alimento y & & 0.57 & \\
\hline & Inga spectabilis (Vahl) Willd. & madera & & 0.07 & \\
\hline & Acacia mangium Willd. & Madera & 1.20 & & \\
\hline & Andira inermis (Sw.) Kunth. & & 0.56 & & 1.88 \\
\hline & Crudia sp 1 & & & & 2.69 \\
\hline & Gliricidia Sepium Kunth ex Steud. & & 0.65 & & \\
\hline & Inga longiflora Spruce ex Benth. & & & 0.20 & \\
\hline & Inga sp 2 & & & 1.46 & \\
\hline & Ormosia nobilis Tul. & & & & 3.79 \\
\hline & Parkia $c f$. nitida Miq. & & & 1.11 & \\
\hline & Platypodium sp 1 & & 0.40 & & \\
\hline \multirow[t]{3}{*}{ Lauraceae } & Persea americana Mill. & Alimento & 6.58 & 0.33 & \\
\hline & Licaria cf. brasiliensis (Nees) Kosterm. & Madera & 0.10 & & \\
\hline & Ocotea tomentosa van der Werff. & & & 1.32 & \\
\hline Lecythidaceae & Cariniana pyriformis Miers. & & 0.93 & & \\
\hline \multirow[t]{2}{*}{ Malvaceae } & Quararibea cordata (Bonpl.) Vischer. & Alimento & 1.90 & & \\
\hline & Pachira sp 1 & Madera & & & 2.28 \\
\hline Meliaceae & Cedrela odorata L. & & 0.03 & & \\
\hline Myrtaceae & Psidium guajava L. & Alimento & 2.18 & 0.02 & \\
\hline \multirow[t]{2}{*}{ Rutaceae } & Citrus reticulata Blanco. & & & 0.11 & \\
\hline & Citrus $x$ sinensis (L.) Osbeck. & & 3.40 & & \\
\hline Sapotaceae & Micropholis sp 1 & Madera & & 0.14 & \\
\hline
\end{tabular}

Olorunfemi et al., 2019), afirmación que concuerda con lo encontrado en el presente estudio (Apéndice digital 1). La mayor acumulación de carbono la presentó el bosque, resultados concordantes con los reportes de Asase \& Tetteh (2015), quienes han encontrado más carbono que en los sistemas productivos. Por ejemplo, en estudios realizados por Nijmeijer et al. (2018) encontraron reservas de carbono arriba del suelo en bosque de $118.4 \mathrm{t} \mathrm{C} \mathrm{ha}^{-1}$, mientras que las sabanas arborizadas acumularon $8 \mathrm{t} \mathrm{C} \mathrm{ha}^{-1}$. En este estudio, la acumulación de carbono fue mayor en la parte aérea, lo que concuerda con Yepes et al. (2015), quienes 
afirman que este reservorio puede contribuir hasta con un $60 \%$ del carbono total. Asase \& Tetteh (2015), indican que, entre las razones de este comportamiento, se encuentra la abundancia de fustales grandes, los cuales almacenan carbono en mayores proporciones; no obstante, en este estudio la mayor acumulación de carbono forestal en bosque, la presentaron las fustales $\left(90 \mathrm{t} \mathrm{C} \mathrm{ha}^{-1}\right)$.

En el presente estudio, la diversidad florística y acumulación de carbono forestal varió en los tres SAF (Apéndice digital), esto ocurre principalmente por aspectos culturales de la zona, el tipo de productor, sus objetivos y conocimiento (Salvador, Cámara, Martínez, Sánchez, \& Valdés, 2019). En el caso de SAF café y SAF cacao, un segundo factor determinante en la composición del dosel es la necesidad de sombra para los arbustos de café y los árboles de cacao (Suárez et al., 2018; Villarreyna, Avelino, \& Cerda, 2020). Estas condiciones son un factor determinante, ya que de acuerdo con lo planteado por Vaast \& Somarriba (2014) y Jiménez et al. (2019), un dosel de especies acompañantes botánicamente diverso y ecológicamente complejo, tiene un impacto positivo en la captura y almacenamiento de carbono.

En el caso de SAF cacao, la acumulación de carbono en la parte forestal presenta similitud con estudios realizados por Salvador et al. (2019) quienes reportaron que las especies de sombra en cacao, acumularon entre 25 y $30 \mathrm{t}$ $\mathrm{C}$ ha ${ }^{-1}$. No obstante, el mismo estudio reportó que los árboles de cacao acumularon entre 6 y 9 t C ha $^{-1}$, lo cual es inferior a lo encontrado en este estudio. Arce, Ortiz, Villalobos, \& Cordero (2008), Andrade, Figueroa, \& Silva (2013) y Marín, Andrade, \& Sandoval (2016), afirman que los árboles de cacao acumularon entre 7 y 38 t C ha $^{-1}$, rangos en los que se encuentran los hallazgos del presente estudio. Por el contrario, Zabala, \& Victorino (2019) estimaron una acumulación de $31.6 \mathrm{t} \mathrm{C} \mathrm{ha}^{-1}$ en árboles de cacao, que representa un $59 \%$ más a lo reportado en esta investigación. Resultados similares fueron encontrados por Saj et al. (2013) quienes reportan $70 \mathrm{t} \mathrm{C} \mathrm{ha}^{-1}$ en especies arbóreas acompañantes al cacao. En SAF café, el carbono acumulado en las especies del dosel de sombra fue inferior a $10 \mathrm{t} \mathrm{C} \mathrm{ha}^{-1}$, lo cual difiere a lo reportado por Vega, Ordoñez, Suárez, \& López (2014), quienes encontraron que las especies del dosel de sombra acumularon entre 34 y $84 \mathrm{t} \mathrm{C} \mathrm{ha}^{-1}$.

El COS fue diferente entre usos del suelo, con los mayores valores encontrados en SAF café y bosque; mientras que el menor fue estimado en SAF cacao. Marques, Gama-Rodrigues, Gama-Rodrigues, \& Bonadie (2016) reportaron valores cercanos a los presentes en el sur de Bahía (Brasil): 31.7, 35.0 y 45.0 t C ha ${ }^{-1}$ en SAF cacao, bosques secundarios y pasturas, respectivamente. Sin embargo, Pocomucha, Alegre, \& Abregú (2016) estimaron en $65.6 \mathrm{t} \mathrm{C}^{-1}$ el COS en SAF cacao en Huánuco, Perú, valor que supera en $56 \%$ a los presentes hallazgos y valores que exceden un $50 \%$ del total de carbono acumulado en los SAF cacao. En SAF café en Veracruz (México), Cristóbal, Tinoco, Prado, \& Hernández (2019) encontraron $76.3 \mathrm{t} \mathrm{C} \mathrm{ha}^{-1}$ como policultivo tradicional y $35.6 \mathrm{t} \mathrm{C} \mathrm{ha}^{-1}$ en policultivo comercial, montos que superan en 44 y $17 \%$ los estimados de este estudio. Alvarado, Andrade, \& Segura (2013), encontraron una acumulación de COS en SAF café con Cordia alliodora Kunth ex Steud. que excede un $24 \%$ lo encontrado en el presente estudio. De acuerdo con Somarriba et al. (2013), la alta acumulación de carbono en suelo bajo SAF se alcanza con un prolongado tiempo de establecimiento, valores que pueden llegar a ser similares a los encontrado en bosques naturales Gama et al. (2010). Esta acumulación tiene estrecha relación con la cobertura vegetal (Cristóbal et al., 2019) y el sistema radicular (Hertel, Harteveld, \& Leuschner, 2009), en especial con la dinámica de las raíces finas (Andrade et al., 2008; Lukac, 2012). Esto es explicado por la cantidad de carbono acumulado en suelo de pasturas, ya que estas presentan una mayor rotación de raíces finas (Guo, Wang, \& Gifford, 2007), resultados que son similares a los reportes de Solly et al. (2013) y Andrade, Espinosa, \& Moreno (2014).

Las especies arbóreas en SAF cacao, SAF café y SSP mejoran la provisión de servicios 
ecosistémicos al remover carbono de la atmósfera y acumularlo por un tiempo prolongado (Andrade \& Ibrahim, 2003; Alvarado et al., 2013; Saj et al., 2013; Wartenberg et al., 2017; Patiño, Suárez, Andrade, \& Segura, 2018; Villa et al., 2020) y, al cumplir funciones como la provisión de sombra, la fertilización del suelo, la producción de frutas y madera (Tschora \& Cherubini, 2020). Esto permite diversificar las dietas alimenticias y los ingresos económicos de los productores (Vaast \& Somarriba, 2014), características que pueden contribuir significativamente a la calidad y los medios de vida de los hogares rurales (Villa et al., 2020).

Persea americana Mill., especie que acumuló el mayor carbono en SAF cacao, presenta un alto y creciente interés económico en Colombia (Pinto, Rueda, \& Arguello, 2019), lo que contribuye a que los SAF potencien su rol de dinamizador de la economía local. La alta acumulación de carbono en especies maderables de alto interés comercial, como Cedrela odorata L., Cordia alliodora Kunth ex Steud. y Cariniana pyriformis Miers., garantiza que después de su aprovechamiento para ebanistería, medios de transporte o construcción, se conserve los contenidos de carbono (Albrecht \& Kandji, 2003).

Las especies que generan material combustible garantiza el suministro de leña utilizada en la cocción de alimentos, lo cual reduce la presión sobre los bosques (De Giusti, Kristjanson, \& Rufino, 2019; Tschora \& Cherubini, 2020) y podrían tener un valor similar al del cultivo principal (Cerda et al., 2014). Sin embargo, esta afirmación sólo es válida parcialmente, ya que la competencia con el cultivo principal puede poner en peligro los beneficios de los SAF (Tschora \& Cherubini, 2020). En tal sentido, varios autores señalan que mayor sombra y diversidad de especies en SAF de cacao y café intensifica el ataque de plagas y enfermedades (Sonwa et al., 2005; Sonwa, Weise, Nkongmeneck, Tchatat, \& Janssens, 2016; Soto \& Jiménez, 2018; Avelino et al., 2020; Hernández et al., 2020), lo que motiva la tumba de especies acompañantes (Vaast \& Somarriba, 2014), disminuyendo las reservas de carbono (Soto \&
Jiménez, 2018). No obstante, Altieri, \& Toledo (2011) afirman que los policultivos reducen las pérdidas generadas por las malezas, insectos y enfermedades, sin embargo, esta afirmación está ligada estrictamente al control natural. En complemento, Hernández et al. (2020) encontraron que, para el caso del cultivo de cacao, cuando el productor hace un adecuado manejo del cultivo, se puede tener un alto dosel de sombra, aumentado los servicios ecosistémicos y teniendo buenos rendimientos de grano de cacao; pero, cuando el productor no hace un adecuado manejo en el cultivo, a mayor sombra, mayor afectación de plagas y enfermedades. En el caso los SPP, las dificultades radican en la dificultad en los procesos de adopción por parte de los productores (Buitrago et al., 2018; Soto \& Jiménez, 2018), dificultades generadas principalmente por el aumento de mano de obra, acciones de planificación y capacitación, además, del alto costo inicial para el establecimiento (Ávila \& Revollo, 2014).

Declaración de ética: los autores declaran que todos están de acuerdo con esta publicación y que han hecho aportes que justifican su autoría; que no hay conflicto de interés de ningún tipo; y que han cumplido con todos los requisitos y procedimientos éticos y legales pertinentes. Todas las fuentes de financiamiento se detallan plena y claramente en la sección de agradecimientos. El respectivo documento legal firmado se encuentra en los archivos de la revista.

\section{AGRADECIMIENTOS}

Los autores agradecen a los hogares rurales del municipio de Mesetas, departamento del Meta, por permitir el ingreso y la toma de datos para la realizaron del presente estudio. A la Universidad de la Amazonia y a la Corporación para el Desarrollo Sostenible del Área de Manejo Especial La Macarena -CORMACARENA-, por la financiación a través del convenio interadministrativo con código PE.GDE.1.4.8.1.19.015 del 07 de junio de 2019. 


\section{RESUMEN}

Introducción: Los cultivos de café, cacao y pasturas para la ganadería son actividades agropecuarias de interés económico en Colombia. Cuando estas actividades se desarrollan bajo sistemas agroforestales (SAF) promueven la conservación e incrementan la fijación de carbono y, por ende, la mitigación del cambio climático. Objetivo: El estudio estimó el almacenamiento de carbono en la biomasa aérea, necromasa y carbono orgánico del suelo bajo SAF con cacao (SAF cacao), SAF con café (café SAF), sistemas silvopastoriles (SSP) y bosque en el municipio de Mesetas, Meta (Colombia). Métodos: Se establecieron 44 parcelas de muestreo, en donde se tomaron medidas dasométricas a individuos con un diámetro del tronco a la altura del pecho (dap) $\geq 2.5 \mathrm{~cm}$ (latizales, fustales y fustales grandes), cuyos valores fueron transformados a carbono con modelos de biomasa y una fracción de carbono default. En los tres sistemas agropecuarios, se contó el número de árboles de cacao, café, plantas asociadas y se identificó el tipo de uso (maderable, alimento, combustión). Resultados: El almacenamiento de carbono presentó diferencias significativas $(\mathrm{P}<0.0001)$ entre usos del suelo. La mayor acumulación se encontró en bosque, con 216.6 t C ha ${ }^{-1}$, superando en 59,72 y $73 \%$ a SAF cacao, SSP y SAF café, respectivamente. Fabaceae, Lauraceae y Primulaceae presentaron el mayor almacenamiento de carbono. En SAF cacao, la mayor acumulación de carbono fue encontrada en especies para alimento humano; en SAF café y SSP, el mayor almacenamiento fue presentado en las especies maderables. Conclusión: Estos resultados resaltan el potencial de almacenamiento de carbono en los sistemas productivos de mayor importancia en el departamento del Meta, lo cual es importante para el diseño de estrategias que permitan integrar acciones de mitigación de emisiones de gases de efecto invernadero y promover la economía campesina local.

Palabras clave: bosques; cambio climático; efecto invernadero; agricultura; ganadería; mitigación.

\section{REFERENCIAS}

Albrecht, A., \& Kandji, S.T. (2003). Carbon sequestration in tropical agroforestry systems. Agriculture, Ecosystems \& Environment, 99(1-3), 15-27. DOI:10.1016/ s0167-8809(03)00138-5

Altieri, M.A., \& Toledo, V.M. (2011). The agroecological revolution in Latin America: rescuing nature, ensuring food sovereignty and empowering peasants. Journal of Peasant Studies, 38(3), 587-612. DOI:10. $1080 / 03066150.2011 .582947$

Alvarado, J., Andrade, H.J., \& Segura, M. (2013). Almacenamiento de carbono orgánico en suelos en sistemas de producción de café (Coffea arabica L.) en el municipio del Líbano, Tolima, Colombia. Colombia Forestal, 16(1), 21-31.
Andrade, H.J., \& Ibrahim, M. (2003). ¿Cómo monitorear el secuestro de carbono en los sistemas silvopastoriles? Agroforestería en las Américas, 10(39), 109-116.

Andrade, H.J., Brook, R., \& Ibrahim, M. (2008). Growth, production and carbon sequestration of silvopastoral systems with native timber species in the dry lowlands of Costa Rica. Plant and Soil, 308(1-2), 11-22. DOI:10.1007/s11104-008-9600-X

Andrade, H.J., Figueroa, J., \& Silva, D. (2013). Almacenamiento de carbono en cacaotales (Theobroma cacao) en armero-guayabal (Tolima, Colombia). Scientia Agroalimentaria, 1, 6-10.

Andrade, H.J., Espinosa, E.L., \& Moreno, H.A. (2014). Impact of grazing on soil organic storage carbon in high lands of Anaime, Tolima, Colombia. Zootecnia Tropical, 32(1), 7-21.

Andrade, H.J., Segura, M.A., Feria, M., \& Suárez, W. (2016). Above-ground biomass models for coffee bushes (Coffea arabica L.) in Líbano, Tolima, Colombia. Agroforestry Systems, 92(3), 775-784. DOI:10.1007/s10457-016-0047-4

Andrade, H.J., Segura, M.A., Canal, D.S., Huertas, A., \& Mosos, C.A. (2017). Composición florística y reservas de carbono en bosques ribereños en paisajes agropecuarios de la zona seca del Tolima, Colombia. Revista de Biología Tropical, 65(4), 1245-1260.

Arce, N., Ortiz, E., Villalobos, M., \& Cordero, S. (2008). Existencias de carbono en charrales y sistemas agroforestales de cacao y banano de fincas indígenas bribri y cabécar de Talamanca, Costa Rica. Agroforestería en las Américas, 46, 30-33.

Arciniegas, S.P., \& Flórez, D.F. (2018). Estudio de los sistemas silvopastoriles como alternativa para el manejo sostenible de la ganadería. Ciencia y Agricultura, 15(2), 107-116. DOI:10.19053/01228420.v15.2

Arias, M.Á., \& Rosales, S. (2019). Educación ambiental y comunicación del cambio climático; Una perspectiva desde el análisis del discurso. Revista Mexicana de Investigación Educativa, 24(80), 247-269.

Arteaga, L.E., \& Burbano, J.E. (2018). Efectos del cambio climático: una mirada al campo. Revista de Ciencias Agrícolas, 35(2), 79-91. DOI:10.22267/ rcia. 183502.93

Asase, A., \& Tetteh, D.A. (2015). Tree diversity, carbon stocks, and soil nutrients in cocoa-dominated and mixed food crops agroforestry systems compared to natural forest in Southeast Ghana. Agroecology and Sustainable Food Systems, 40(1), 96-113. DOI:10.10 $80 / 21683565.2015 .1110223$

Ávila, V.S., \& Revollo, D. (2014). Análisis financiero y percepción de los servicios ambientales de un sistema silvopastoril: un estudio de caso en los Tuxtlas, 
México. Revista Iberoamericana de Economía Ecológica, 22, 17-33.

Baena, J.J. (2019). La política de comercio exterior y las exportaciones colombianas. Revista de Economía Institucional, 21(41), 51-70. DOI:10.18601/01245996. v $2 \ln 41.03$

Buitrago, M.E., Ospina, L.A., \& Narváez, W. (2018). Sistemas Silvopastoriles: alternativa en la mitigación y adaptación de la producción bovina al cambio climático. Boletín Científico. Centro de Museos. Museo de Historia Natural, 22(1), 31-42. DOI:10.17151/ bccm.2018.22.1.2

Casanoves, F., Cifuentes, M., \& Chacón, M. (2017). Estimación del carbono a partir de inventarios forestales nacionales Buenas prácticas para la recolección, manejo y análisis de datos. Turrialba, Costa Rica: Centro Agronómico Tropical de Investigación y enseñanza, CATIE.

Cerda, R., Deheuvels, O., Calvache, D., Niehaus, L., Saenz, Y., Kent, J., Vilchez, S., Villota, A., Martinez, C., \& Somarriba, E. (2014) Contribution of cocoa agroforestry systems to family income and domestic consumption: looking toward intensification. Agroforestry Systems, 88: 957-981 DOI:10.1007/ s10457-014-9691-8

Chave, J., Andalo, C., Brown, S., Cairns, M.A., Chambers, J.Q., Eamus, D., . . . Yamakura, T. (2005). Tree allometry and improved estimation of carbon stocks and balance in tropical forests. Oecologia, 145(1), 87-99. DOI:10.1007/s00442-005-0100-x

Cristóbal, D., Tinoco, J.Á., Prado, J.V., \& Hernández, E. (2019). Soil carbon and nitrogen in tropical montane cloud forest, agroforestry and coffee monoculture systems. Revista Chapingo Serie Ciencias Forestales y del Ambiente, 25(2), 169-184. DOI:10.5154/r. rchscfa.2018.09.070

De Giusti, G., Kristjanson, P., \& Rufino, M.C. (2019). Agroforestry as a climate change mitigation practice in smallholder farming: evidence from Kenya. Climatic Change, 153(3), 379-394. DOI:10.1007/ s10584-019-02390-0

Delgado, H., Rangel, J.A., \& Silva, A. (2018). Caracterización de la fertilidad química de los duelos en sistemas productivos de la altillanura plana, Meta, Colombia. Luna Azul, (46), 54-69. DOI:10.17151/ luaz.2018.46.5

Di Rienzo, J., Macchiavelli, R., \& Casanoves, F. (2011). Modelos lineales mixtos: aplicaciones en InfoStat (1 $1^{\text {era }}$ Ed.). Córdoba, Argentina: Grupo InfoStat.

Di Rienzo, J., Casanoves, F., Balzarini, M., Gonzalez, L., Tablada, M., \& Robledo, C. (2019). Infostat (Version 2019). Recuperado de http://www.infostat.com.ar
Díaz, M.F., Enciso, K., Triana, N., Muriel, J., \& Burkart, S. (2018). Pagos por Servicios Ambientales para sistemas silvopastoriles en Colombia. Centro Internacional de Agricultura Tropical (CIAT), Cali, CO. 54 p. Recuperado de https:/www.ilri.org/publications/pagos-por-servicios-ambientales-para-sistemassilvopastoriles-en-colombia

Espinosa, J.A. (2016). Características estructurales y funcionales de un faro agroecológico a partir de las experiencias de productores cacaoteros de las regiones de los departamentos de Nariño, Meta, Caquetá y Tolima (Tesis de Doctorado). Universidad de Antioquia, Medellín, Colombia. Recuperado de http://hdl. handle.net/10495/5644

Gama, E.F., Ramachandran, P.K., Nair, V.D., Gama, A.C., Baligar, V.C., \& Machado, R.C. (2010). Carbon storage in soil size fractions under two cacao agroforestry systems in Bahia, Brazil. Environmental Management, 45(2), 274-283. DOI:10.1007/ s00267-009-9420-7

Gerber, P., Steinfeld, H., \& Henderson, B. (2013). Tackling climate change through livestock - A global assessment of emissions and mitigation opportunities. Roma, Italia: Food and Agriculture Organization of the United Nations (FAO).

Goodman, R.C., Phillips, O.L., Del Castillo Torres, D., Freitas, L., Cortese, S.T., Monteagudo, A., \& Baker, T.R. (2013). Amazon palm biomass and allometry. Forest Ecology and Management, 310, 994-1004. DOI:10.1016/j.foreco.2013.09.045

Guo, L.B., Wang, M., \& Gifford, R.M. (2007). The change of soil carbon stocks and fine root dynamics after land use change from a native pasture to a pine plantation. Plant and Soil, 299(1-2), 251-262. DOI:10.1007/s11104-007-9381-7

Gutiérrez, G., Gutiérrez-Montes, I., Hernández-Núñez, H.E., Suárez, J.C., \& Casanoves, F. (2020). Relevance of local knowledge in decision-making and rural innovation: A methodological proposal for leveraging participation of Colombian cocoa producers. Journal of Rural Studies, 75, 119-124. DOI:10.1016/j. jrurstud.2020.01.012

Hernández, H.E., Gutiérrez-Montes, I., Gutiérrez, G.A., Sánchez, J., Suárez, J.C., \& Casanoves, F. (2020). Agronomic state of cacao cultivation: its relationship with the capitals endowment of the Colombian rural households. Agroforestry Systems. DOI:10.1007/ s10457-020-00556-9

Hertel, D., Harteveld, M.A., \& Leuschner, C. (2009). Conversion of a tropical forest into agroforest alters the fine root-related carbon flux to the soil. Soil Biology and Biochemistry, 41(3), 481-490. DOI:10.1016/j. soilbio.2008.11.020

Hurtado, C., Corte, C., \& Triana, M. (2017). Estimación del carbono almacenado en el bosque natural en la 
cuenca media-baja. municipio de río Quito Chocó, Colombia. Ingeniería e Innovación, 5(1), 32-42. DOI: $10.21897 / 23460466.1102$

IDEAM. (2010). Leyenda nacional de coberturas de la tierra. Metodología CORINE Land Cover adaptada para Colombia, escala 1:100.000. Bogotá, Bogotá, D.C: Instituto de Hidrología, Meteorología y Estudios Ambientales -IDEAM-.

IDEAM. (2017). Atlas climatológico de Colombia. Bogotá, D.C., Colombia: Instituto de Hidrología, Meteorología y Estudios Ambientales - IDEAM.

IDEAM. (2018). Manual de Campo: Inventario Forestal Nacional de Colombia. Bogotá.

IGAC. (2006). Métodos analíticos del laboratorio de suelos (6 $6^{\text {ta }}$ Ed.). Bogotá, Colombia.

IPCC. (2000). Good Practice Guidance and Uncertainty Management in National Greenhouse Gas Inventories. IPCC National Greenhouse Gas Inventories Programme. Japan: Institute for Global Environmental Strategies.

IPCC. (2006). IPCC Guidelines for National Greenhouse Gas Inventories. H.S. Eggleston, K. Miwa, N. Srivastava, \& K. Tanabe (Eds.). Tokyo, Japan: National Greenhouse Gas Inventories Programme.

IPCC. (2019). Climate change and land. An IPCC special report on climate change, desertification, land degradation, sustainable land management, food security, and greenhouse gas fluxes in terrestrial ecosystems. Summary for policymakers. Retrieved from https:// www.ipcc.ch/site/assets/uploads/2019/11/SRCCLFull-Report-Compiled-191128.pdf

Jagoret, P., Michel, I., Ngnogué, H.T., Lachenaud, P., Snoeck, D., \& Malézieux, E. (2017). Structural characteristics determine productivity in complex cocoa agroforestry systems. Agronomy for Sustainable Development, 37(6). DOI:10.1007/ s13593-017-0468-0

Jiménez, E.R., Fonseca, W., \& Pazmiño, L. (2019). Sistemas Silvopastoriles y Cambio climático: Estimación y predicción de Biomasa Arbórea. La Granja, 29(1), 44-55. DOI:10.17163/lgr.n29.2019.04

Kuosmanen, T., Zhou, X., \& Dai, S. (2020). How much climate policy has cost for OECD countries? World Development, 125, 104681. DOI:10.1016/j. worlddev.2019.104681

Lizcano, R., Olivera, D., Saavedra, D., Machado, L., Rolando, E., Moreno, M.F., \& Flórez, M.F. (2017) Muestreo de suelos, técnicas de laboratorio e interpretación de análisis de suelos. Neiva-Huila, Colombia: Centro de Formación Agroindustrial La Angostura Servicio Nacional de Aprendizaje (SENA).
López, J.G., Casanova, F., Villanueva, G., Díaz, V.F., Solorio, F.J., Martínez, P., . . Chay-Canul, A.J. (2018). Carbon storage in a silvopastoral system compared to that in a deciduous dry forest in Michoacán, Mexico. Agroforestry Systems, 93(1), 199-211. DOI:10.1007/ s10457-018-0259-x

Lukac, M. (2012) Fine Root Turnover. In S. Mancuso (Ed.), Measuring Roots: an updated approach. (pp. 363-373). Berlin Heidelberg: Springer-Verlag. DOI: 10.1007/978-3-642-22067-8 18

Marín, M.D.P., Andrade, H.J., \& Sandoval, A.P. (2016). Fijación de carbono atmosférico en la biomasa total de sistemas de producción de cacao en el departamento del Tolima, Colombia. Revista U.D.C.A Actualidad \& Divulgación Científica, 19(2), 351-360.

Marques, P.H., Gama-Rodrigues, E.F., Gama-Rodrigues, A.C., \& Bonadie, J.R. (2016). Soil carbon stocks and origin under different cacao agroforestry systems in Southern Bahia, Brazil. Agriculture, Ecosystems \& Environment, 221, 99-108. DOI:10.1016/j. agee.2016.01.022

Ngo Bieng, M.A., Gidoin, C., Avelino, J., Cilas, C., Deheuvels, O., \& Wery, J. (2013). Diversity and spatial clustering of shade trees affect cacao yield and pathogen pressure in Costa Rican agroforests. Basic and Applied Ecology, 14(4), 329-336. DOI:10.1016/j. baae.2013.03.003

Nielsen, T.D. (2016). From REDD+ forests to green landscapes? Analyzing the emerging integrated landscape approach discourse in the UNFCCC. Forest Policy and Economics, 73, 177-184. DOI:10.1016/j. forpol.2016.09.006

Nijmeijer, A., Lauri, P.É., Harmand, J.M., \& Saj, S. (2018). Carbon dynamics in cocoa agroforestry systems in Central Cameroon: afforestation of savannah as a sequestration opportunity. Agroforestry Systems, 93(3), 851-868. DOI:10.1007/s10457-017-0182-6

Olorunfemi, I.E., Komolafe, A.A., Fasinmirin, J.T., \& Olufayo, A.A. (2019). Biomass carbon stocks of different land use management in the forest vegetative zone of Nigeria. Acta Oecologica, 95, 45-56. DOI:10.1016/j. actao.2019.01.004

Paipa, N.A., \& Triana, M.A. (2018). Estimación del carbono almacenado en la biomasa aérea de un bosque húmedo tropical en Paimadó, Chocó. Ingenierías USBMed, 9(1), 18-29. DOI:10.21500/20275846.3180

Patiño, S., Suárez, L., Andrade, H.J., \& Segura, M.A. (2018). Captura de carbono en biomasa en plantaciones forestales y sistemas agroforestales en Armero-Guayabal, Tolima, Colombia. Revista de Investigación Agraria y Ambiental, 9(2), 121-133. DOI:10.22490/21456453.2312

Pinto, J., Rueda, H., \& Arguello, H. (2019). Classification of Hass avocado (persea americana mill) in terms of 
its ripening via hyperspectral images. TecnoLógicas, 22(45), 109-128. DOI:10.22430/22565337.1232

Pocomucha, V.S., Alegre, J., \& Abregú, L. (2016). Análisis socio económico y carbono almacenado en sistemas agroforestales de cacao (Theobroma cacao L.) en Huánuco. Ecología Aplicada, 15(2), 107-114. DOI:10.21704/rea.v15i2.750

Ricker, M. (2019). Manual para realizar las colectas botánicas del Inventario Nacional Forestal y de Suelos de México. Ciudad de México: Instituto de Biología, Universidad Nacional Autónoma de México.

Rojas, M.M., Nejadhashemi, A.P., Harrigan, T., \& Woznicki, S.A. (2017). Climate change and livestock: Impacts, adaptation, and mitigation. Climate Risk Management, 16, 145-163. DOI:10.1016/j.crm.2017.02.001

Saj, S., Jagoret, P., \& Todem, H. (2013). Carbon storage and density dynamics of associated trees in three contrasting Theobroma cacao agroforests of Central Cameroon. Agroforestry Systems, 87(6), 1309-1320. DOI:10.1007/s10457-013-9639-4

Salvador, P., Cámara, L.D.C., Martínez, J.L., Sánchez, R., \& Valdés, E. (2019). Diversidad, estructura y carbono de la vegetación arbórea en sistemas agroforestales de cacao. Madera y Bosques, 25(1). DOI:10.21829/ myb.2019.2511638

Segura, M.A., Andrade, H.J., \& Mojica Sánchez, C.A. (2019). Estructura, composición florística y almacenamiento de carbono en bosques nativos del páramo de Anaime, Tolima, Colombia. Ciência Florestal, 29(1), 157-168. DOI:10.5902/1980509826551

Segura, M.A., Andrade, H.J., \& Sierra, E. (2020). Diversidad florística y captura de carbono en robledales y pasturas con árboles en Santa Isabel, Tolima, Colombia. Revista de Biología Tropical, 68(2), 383-393.

Smiley, G.L., \& Kroschel, J. (2008). Temporal change in carbon stocks of cocoa-gliricidia agroforests in Central Sulawesi, Indonesia. Agroforestry Systems, 73(3), 219-231. DOI:10.1007/s10457-008-9144-3

Solly, E., Schöning, I., Boch, S., Müller, J., Socher, S.A., Trumbore, S.E., \& Schrumpf, M. (2013). Mean age of carbon in fine roots from temperate forests and grasslands with different management. Biogeosciences, 10(7), 4833-4843. DOI:10.5194/ bg-10-4833-2013

Somarriba, E., Cerda, R., Orozco, L., Cifuentes, M., Dávila, H., Espin, T., . . . Deheuvels, O. (2013). Carbon stocks and cocoa yields in agroforestry systems of Central America. Agriculture, Ecosystems \& Environment, 173, 46-57. DOI:10.1016/j.agee.2013.04.013

Sonwa, D.J., Weise, S., Adesina, A., Nkongmeneck, A.B., Tchatat, M., \& Ndoye, O. (2005). Production constraints on cocoa agroforestry systems in West and Central Africa: The need for integrated pest management and multi-institutional approaches. Forestry Chronicle, 81(3), 345-349. DOI:10.5558/tfc81345-3

Sonwa, D.J., Weise, S.F., Nkongmeneck, B.A., Tchatat, M., \& Janssens, M.J.J. (2016). Structure and composition of cocoa agroforests in the humid forest zone of Southern Cameroon. Agroforestry Systems, 91(3), 451-470. DOI:10.1007/s10457-016-9942-y

Soto-Pinto, L., Anzueto, M., Mendoza, J., Ferrer, G.J., \& de Jong, B. (2010). Carbon sequestration through agroforestry in indigenous communities of Chiapas, Mexico. Agroforestry Systems, 78(1), 39-51. DOI:10.1007/s10457-009-9247-5

Soto, L., \& Jiménez, G. (2018). Contradicciones socioambientales en los procesos de mitigación, asociados al ciclo del carbono en sistemas agroforestales. Madera y Bosques, 24, e2401887. DOI:10.21829/ myb.2018.2401887

Suárez, J.C., Ngo Bieng, M.A., Melgarejo, L.M., Di Rienzo, J.A., \& Casanoves, F. (2018). First typology of cacao (Theobroma cacao L.) systems in Colombian Amazonia, based on tree species richness, canopy structure and light availability. PLoS One, 13(2), e0191003. DOI:10.1371/journal.pone.0191003

Sullivan, M.J., Talbot, J., Lewis, S.L., Phillips, O.L., Qie, L., Begne, S.K., . . . Zemagho, L. (2017). Diversity and carbon storage across the tropical forest biome. Scientific Reports, 7, 39102. DOI:10.1038/srep39102

Tschora, H., \& Cherubini, F. (2020). Co-benefits and tradeoffs of agroforestry for climate change mitigation and other sustainability goals in West Africa. Global Ecology and Conservation, 22. DOI:10.1016/j. gecco.2020.e00919

Vaast, P., \& Somarriba, E. (2014). Trade-offs between crop intensification and ecosystem services: the role of agroforestry in cocoa cultivation. Agroforestry Systems, 88(6), 947-956. DOI:10.1007/ s10457-014-9762-x

Vega, G., Ordoñez, C.M., Suárez, J.C., \& López, C.F. (2014). Almacenamiento de carbono en arreglos agroforestales asociados con café (Coffea arabica) en el sur de Colombia. Revista de Investigación Agraria y Ambiental, 5(1), 213-221.

Villa, P.M., Martins, S.V., de Oliveira Neto, S.N., Rodrigues, A.C., Hernández, E.P., \& Kim, D.G. (2020). Policy forum: Shifting cultivation and agroforestry in the Amazon: Premises for REDD+. Forest Policy and Economics, 118. DOI:10.1016/j.forpol.2020.102217

Villarreyna, R.A., Avelino, J., \& Cerda, R. (2020). Adaptación basada en ecosistemas: efecto de los árboles de sombra sobre servicios ecosistémicos en cafetales. Agronomía Mesoamericana, 499-516. DOI:10.15517/am.v31i2.37591 
Wartenberg, A.C., Blaser, W.J., Gattinger, A., Roshetko, J.M., Van, M., \& Six, J. (2017). Does shade tree diversity increase soil fertility in cocoa plantations? Agriculture, Ecosystems \& Environment, 248, 190199. DOI:10.1016/j.agee.2017.07.033

Yepes, A.P., Navarrete, D.A., Duque, A.J., Phillips, J.F., Cabrera, K.R., Álvarez, E., . . . Ordoñez, M.F. (2011). Protocolo para la estimación nacional y subnacional de biomasa - carbono en Colombia. Bogotá D.C., Colombia: Instituto de Hidrología, Meteorología, y Estudios Ambientales -IDEAM-.

Yepes, A., Herrera, J., Phillips, J., Cabrera, E., Galindo, G., Granados, E., . . . Cardona, M. (2015). Contribución de los bosques tropicales de montaña en el almacenamiento de carbono en Colombia. Revista de Biología Tropical, 63(1), 69-82.

Zabala, F.A., \& Victorino, I. (2019). Capacidad adaptativa y vulnerabilidad de la cuenca del río Orotoy ante el cambio climático, a partir del análisis de las variables de los medios de vida. Biodiversidad en la Práctica, 4(1), 51-85.

Zapata, P.C. (2019). Composición y estructura del dosel de sombra en sistemas agroforestales con café de tres municipios de Cundinamarca, Colombia. Ciência Florestal, 29(2), 685-697. DOI:10.5902/1980509827037

See Digital Appendix at: / Ver Apéndice digital en: revistas.ucr.ac.cr 